TAIWANESE JOURNAL OF MATHEMATICS

Vol. 14, No. 2, pp. 501-515, April 2010

This paper is available online at http://www.tjm.nsysu.edu.tw/

\title{
ON DOMINATING PHENOMENON FOR BOUNDED REAL HARMONIC FUNCTIONS IN SEVERAL VARIABLES
}

\author{
So-Chin Chen* and Cin-Chang Lu
}

\begin{abstract}
In this article, motivated by the work of Brown, Shields, and Zeller[2], we first consider the representation problem of zero by exponential sums in several complex variables. Then we give a complete characterization of dominating sets for bounded real harmonic functions, denoted by $h^{\infty}\left(\Re B_{n}\right)$, on the open unit ball $\Re B_{n}$ in $\mathbb{R}^{n}, n \geq 3$, and for bounded real $\mathcal{M}$-harmonic functions, denoted by $\mathcal{M} h^{\infty}\left(B_{n}\right)$, on the open unit ball $B_{n}$ in $\mathbb{C}^{n}, n \geq 2$.
\end{abstract}

\section{INTRODUCTION}

In this article, motivated by the work of Brown, Shields, and Zeller[2], we shall first consider the following representation problem of zero by exponential sums in several complex variables. Namely, is it possible to find a sequence of distinct points $\left\{\alpha_{k}\right\}$ from $B_{n}$ and a sequence of complex numbers $\left\{a_{k}\right\}$ such that

$$
0 \equiv \sum_{k=1}^{\infty} a_{k} e^{\alpha_{k} z}, \quad \text { and } \quad 0<\sum_{k=1}^{\infty}\left|a_{k}\right|<\infty .
$$

Here $B_{n}=\left\{z \in \mathbb{C}^{n}|| z \mid<1\right\}$ is the open unit ball in $\mathbb{C}^{n}, n \geq 2$, and $S=\partial B_{n}$ denote the boundary of $B_{n}$. We have adopted the notations $\alpha_{k}=$ $\left(\alpha_{k 1}, \cdots, \alpha_{k n}\right), z=\left(z_{1}, \cdots, z_{n}\right)$ and $\alpha_{k} z=\sum_{j=1}^{n} \alpha_{k j} z_{j}$. Denote by $\mathcal{O}\left(B_{n}\right)$ the space of holomorphic functions on $B_{n}$, and let

$$
H^{\infty}\left(B_{n}\right)=\left\{f \in \mathcal{O}\left(B_{n}\right)\left|\|f\|_{\infty}=\sup _{z \in B_{n}}\right| f(z) \mid<\infty\right\} .
$$

Received July 22, 2008, accepted August 6, 2008.

Communicated by J. C. Yao.

2000 Mathematics Subject Classification: 32A30, 30D10.

Key words and phrases: Dominating sets, Harmonic functions, $\mathcal{M}$-harmonic functions.

The authors are partially supported by the grant NSC 96-2115-M-007-005 from the National Science Council of the Republic of China.

*Corresponding author. 
Denote also by $E\left(B_{n}\right)$ the space of all entire functions $g(z)$ that admit a representation of the form

$$
g(z)=\sum_{k=1}^{\infty} c_{k} e^{\beta_{k} z}
$$

with $\sum_{k=1}^{\infty}\left|c_{k}\right|<\infty$ and $\beta_{k} \in B_{n}$ for all $k$.

Obviously, $E\left(B_{n}\right)$ is a complex vector space. Define a norm on $E\left(B_{n}\right)$ by

$$
\|g\|_{E}=\inf \sum_{k=1}^{\infty}\left|c_{k}\right|
$$

where the infimum is taken over all possible representations (1.2) of $g$.

Definition 1.4. A subset $E$ of $B_{n}$ is said to be nontangentially dense almost everywhere on $S$ if almost every boundary point $\zeta \in S$ can be approached nontangentially by a subsequence of points of $E$.

When $n=1$, Brown, Shields, and Zeller showed in [2] the following theorem.

Theorem 1.5. Suppose $\left\{\omega_{k}\right\}$ is a sequence of distinct points in $B_{1}=U$ with no interior limit points, then the following four statements are equivalent:

(i) $\left\{\omega_{k}\right\}$ is nontangentially dense almost everywhere on $\mathbb{T}=\partial U$.

(ii) $\|f\|_{\infty}=\sup _{k}\left|f\left(\omega_{k}\right)\right|$ for every $f \in H^{\infty}(U)$.

(iii) $\left\{\omega_{k}\right\}$ represents all of $E(U)$, i.e., if $g \in E(U)$ and $\epsilon>0$ are given, then there exists $\left\{a_{k}\right\} \in l^{1}$ such that

$$
g(z)=\sum_{k=1}^{\infty} a_{k} e^{\omega_{k} z}, \sum_{k=1}^{\infty}\left|a_{k}\right|<\|g\|_{E}+\epsilon .
$$

(iv) $\left\{\omega_{k}\right\}$ represents zero, i.e., there exists $\left\{a_{k}\right\} \in l^{1}$, not all zero, such that

$$
0=\sum_{k=1}^{\infty} a_{k} e^{\omega_{k} z}
$$

If the sequence of points $\left\{\omega_{k}\right\}$ has limit points in $U$, Theorem 1.5 still hold. See also Bonsall[1] for related results.

For $n \geq 2$, we may introduce another concept of approaching the boundary points, namely, the $K$-limit which is more general than the nontangential approach. See Section 2 for the details. Then we prove the following theorem in several complex variables.

Theorem 1.7. Let $\left\{\alpha_{k}\right\}_{k=1}^{\infty}$ be a sequence of distinct points in $B_{n}, n \geq 2$, with no interior limit points. Then we have (i) $\Rightarrow($ ii $) \Rightarrow($ iii $) \Rightarrow($ iv $)$, where 
(i) $\left\{\alpha_{k}\right\}$ is $K$-dense almost everywhere on $S$.

(ii) $\|f\|_{\infty}=\sup _{k}\left|f\left(\alpha_{k}\right)\right|$ for all $f \in H^{\infty}\left(B_{n}\right)$.

(iii) $\left\{\alpha_{k}\right\}$ represents all of $E\left(B_{n}\right)$, i.e., if $g \in E\left(B_{n}\right)$ and $\epsilon>0$ are given, then there exists coefficients $\left\{a_{k}\right\}$ such that

$$
g(z)=\sum_{k=1}^{\infty} a_{k} e^{\alpha_{k} z}, \quad \text { and } \quad \sum_{k=1}^{\infty}\left|a_{k}\right|<\|g\|_{E}+\epsilon .
$$

(iv) $\left\{\alpha_{k}\right\}$ represents zero, i.e., there exists an element $\left(a_{1}, a_{2}, \cdots\right) \in l^{1}$, not all zero, such that (1.1) holds. Also, the projected sequence of $\left\{\alpha_{k}\right\}$ in every complex line $\mathbb{L}$ through the origin is nontangentially dense on $\mathbb{T}=\partial U$, where $U$ is the open unit disc in $\mathbb{L}$.

Obviously, (iv) does not imply $(i i)$. For instance, if we let $\alpha_{k}=\left(\omega_{k}, 0\right) \in B_{2}$ such that $\left\{\omega_{k}\right\}$ is nontangentially dense almost everywhere on $\mathbb{T}$ in $z_{1}$ component, then $(i v)$ of Theorem 1.5 implies $\left\{\alpha_{k}\right\}$ represents zero in $B_{2}$. But, for the function $f\left(z_{1}, z_{2}\right)=z_{2}$, we have $\|f\|_{\infty, B_{2}}=1>0=\sup _{k}\left|f\left(\alpha_{k}\right)\right|$.

Also, it was shown in Massaneda and Thomas[7] that, if $n>1$, there exists a sequence of points $\left\{\alpha_{k}\right\}_{k=1}^{\infty}$ in $B_{n}$ satisfying $\|f\|_{\infty}=\sup _{k}\left|f\left(\alpha_{k}\right)\right|$ for all $f \in$ $H^{\infty}\left(B_{n}\right)$, but the $K$-limit set of $\left\{\alpha_{k}\right\}$ on $S$ is of Lebesgue measure zero. Therefore, (ii) fails to imply $(i)$ either.

In general, it is still not clear that, if $n \geq 2$, what condition on the sequences of points $\left\{\alpha_{k}\right\}$ really characterizes the statements $(i i)$ or $(i i i)$ or $(i v)$.

Therefore, we consider in Section 3 a similar problem for the space of bounded real harmonic functions, denoted by $h^{\infty}\left(\Re B_{n}\right)$, on the open unit ball $\Re B_{n}$ in $\mathbb{R}^{n}$, $n \geq 3$. We also make the following definition.

Definition 1.9. A subset $E$ of $\Re B_{n}$ is said to be a dominating set for $h^{\infty}\left(\Re B_{n}\right)$ if for every two functions $f, g \in h^{\infty}\left(\Re B_{n}\right)$ satisfying $|f(x)| \leq|g(x)|, x \in E$, we have $\|f\|_{\infty} \leq\|g\|_{\infty}$.

Then we prove the theorem.

Theorem 1.10. Let $\left\{\alpha_{k}\right\}_{k=1}^{\infty}$ be a sequence of distinct points in $\Re B_{n}, n \geq 3$, with no interior limit points. Then the following three statements are equivalent:

(i) $\left\{\alpha_{k}\right\}$ is nontangentially dense almost everywhere on $S=\partial \Re B_{n}$.

(ii) $\|f\|_{\infty}=\sup _{k}\left|f\left(\alpha_{k}\right)\right|$ for every $f \in h^{\infty}\left(\Re B_{n}\right)$.

(iii) $\left\{\alpha_{k}\right\}$ is a dominating set for $h^{\infty}\left(\Re B_{n}\right)$. 
Finally, we consider in Section 4 the same problem for the space of bounded real $\mathcal{M}$-harmonic functions, denoted by $\mathcal{M} h^{\infty}\left(B_{n}\right)$, on the open unit ball $B_{n}$ in $\mathbb{C}^{n}, n \geq 2$. The concept of dominating set can also be introduced for $\mathcal{M} h^{\infty}\left(B_{n}\right)$. Then we prove a similar theorem as follows.

Theorem 1.11. Let $\left\{\alpha_{k}\right\}_{k=1}^{\infty}$ be a sequence of distinct points in $B_{n}, n \geq 2$, with no interior limit points. Then the following three statements are equivalent:

(i) $\left\{\alpha_{k}\right\}$ is $K$-dense almost everywhere on $S$.

(ii) $\|f\|_{\infty}=\sup _{k}\left|f\left(\alpha_{k}\right)\right|$ for every $f \in \mathcal{M} h^{\infty}\left(B_{n}\right)$.

(iii) $\left\{\alpha_{k}\right\}$ is a dominating set for $\mathcal{M} h^{\infty}\left(B_{n}\right)$.

The following corollary can also be obtained immediately via the concept of dominating sets for $H^{\infty}\left(B_{n}\right)$.

Corollary 1.12. Let $E$ be a subset of $B_{n}, n \geq 2$. Then $E$ is a dominating set for $H^{\infty}\left(B_{n}\right)$ if and only if $\|f\|_{\infty}=\sup _{z \in E}|f(z)|$ for every $f \in H^{\infty}\left(B_{n}\right)$.

For related results concerning the dominating sets see Chen[3] for uniform algebra on pseudoconvex domains, Danikas and Hayman[4] and Hayman[5] for the Hardy space $H^{p}(U)$ on the open unit disc $U$ in $\mathbb{C}$.

\section{MAIN Results}

In this section we will prove Theorem 1.7. Most of the ideas in this section are motivated by the work of Brown, Shields, and Zeller[2]. For self-containedness we shall supply a complete proof of the theorem. Recall that $n \geq 2$ throughout this section. First we make the following definitions.

Definition 2.1. Let $\mu$ be a complex Borel measure of compact support $K$ in $\mathbb{C}^{n} . \mu$ is said to represent zero if

$$
\int_{\mathbb{C}^{n}} e^{z w} d \mu(w)=0, z \in \mathbb{C}^{n}
$$

Definition 2.2. A complex Borel measure $\mu$ is said to be concentrated on $B_{n}$ if $\mu(E)=0$ for all subsets $E$ with $E \cap B_{n}=\emptyset$.

Note that the notion stated in Definition 2.2 is somewhat different from saying that the support of $\mu$ is contained in $B_{n}$. 
Again, we agree that $z w=\sum_{j=1}^{n} z_{j} w_{j}, z, w \in \mathbb{C}^{n}$. For a multiindex $m=$ $\left(m_{1}, \cdots, m_{n}\right), m_{j} \in\{0\} \cup \mathbb{N}$, denote the length of $m$ by $|m|=m_{1}+\cdots+m_{n}$, and by $z^{m}=z_{1}^{m_{1}} \cdots z_{n}^{m_{n}}$. Also, the operator $\frac{\partial^{|m|}}{\partial z^{m}}$ stands for $\frac{\partial^{|m|}}{\partial z_{1}^{m_{1}} \cdots \partial z_{n} m_{n}}$.

Then we have

Lemma 2.3. Let $\mu$ be a complex Borel measure concentrated on $B_{n}$. Then the following three statement are equivalent.

$$
\int_{\mathbb{C}^{n}} e^{z w} d \mu(w)=0, z \in \mathbb{C}^{n}
$$

$$
\int_{B_{n}} f(w) e^{z w} d \mu(w)=0, \quad \text { for all } f \in H^{\infty}\left(B_{n}\right), \quad z \in \mathbb{C}^{n}
$$

$$
\int_{B_{n}} f(w) d \mu(w)=0, \quad \text { for all } f \in H^{\infty}\left(B_{n}\right)
$$

Proof. $\quad(2) \Rightarrow(3)$ is trivial by setting $z=0 .(3) \Rightarrow(1)$ is also obvious, since $e^{z w} \in H^{\infty}\left(B_{n}\right)$ for each $z \in \mathbb{C}^{n}$. Thus, we only need to prove (1) implies (2).

Suppose (1) holds. Let $m=\left(m_{1}, \cdots, m_{n}\right)$ be a multiindex. Then

$$
0=\frac{\partial^{|m|}}{\partial z^{m}} \int_{\mathbb{C}^{n}} e^{z w} d \mu(w)=\int_{\mathbb{C}^{n}} w^{m} e^{z w} d \mu(w) .
$$

Thus, (2) holds if $f(w)$ is a polynomial. For any $f \in H^{\infty}\left(B_{n}\right)$, let $f_{\rho}(z)=f(\rho z)$ for $0<\rho<1$. Then $f_{\rho}$ is holomorphic in some open neighborhood of $\bar{B}_{n}$, and $f_{\rho}$ can be approximated uniformly on $\bar{B}_{n}$ by polynomials. Hence, (2) holds for $f_{\rho}$.

Finally, for any $f \in H^{\infty}\left(B_{n}\right)$, using Lebesgue dominated convergence theorem we obtain

$$
\int_{B_{n}} f(w) e^{z w} d \mu(w)=\lim _{\rho \rightarrow 1^{-}} \int_{B_{n}} f_{\rho}(w) e^{z w} d \mu(w)=0 .
$$

This proves (2), and hence the lemma.

Next, we recall the concept of $K$-limit. For $\alpha>1$ and $\zeta \in S$, let

$$
D_{\alpha}(\zeta)=\left\{z \in \mathbb{C}^{n}|| 1-\langle z, \zeta\rangle \mid<\frac{\alpha}{2}\left(1-|z|^{2}\right)\right\} .
$$

Here $\langle z, \zeta\rangle=\sum_{j=1}^{n} z_{j} \bar{\zeta}_{j}$. It is clear that $D_{\alpha}(\zeta) \subset B_{n}$ and $D_{\alpha}(\zeta)=\emptyset$ if $\alpha \leq 1$. Also, for every fixed $\zeta \in S$, the region $D_{\alpha}(\zeta)$ fill $B_{n}$ as $\alpha \rightarrow \infty$. One should note that a sequence of points in $D_{\alpha}(\zeta)$ may approach the boundary point $\zeta$ tangentially. 
Definition 2.4. Let $\zeta \in S$. A function $F: B_{n} \rightarrow \mathbb{C}$ is said to have $K$-limit $\lambda$ at $\zeta$, denoted by $(K-\lim F)(\zeta)=\lambda$, if for every $\alpha>1$ and every sequence $\left\{\mathrm{z}_{k}\right\}$ in $D_{\alpha}(\zeta)$ that converges to $\zeta, F\left(z_{k}\right) \rightarrow \lambda$ as $k \rightarrow \infty$.

Definition 2.5. A subset $E$ of $B_{n}$ is said to be $K$-dense almost everywhere on $S$ if almost every boundary point $\zeta \in S$ can be approached by a sequence of points of $E$ within some $D_{\alpha}(\zeta)$.

In order to prove Theorem 1.7, we also need the following theorem from functional analysis, for instance, see Hille and Phillips[6].

Theorem 2.6. Let $X$ be a Banach space, and let $X^{*}$ be its adjoint space. Suppose $\Sigma$ is a weak ${ }^{*}$-closed subspace of $X^{*}$ and $x_{0}^{*} \notin \Sigma$. Then for each $M$ satisfying the condition $0<M<\inf \left\{\left\|x^{*}-x_{0}^{*}\right\|: x^{*} \in \Sigma\right\}$, there exists an $x_{0} \in X$ such that

$$
x_{0}^{*}\left(x_{0}\right)=1, \quad x^{*}\left(x_{0}\right)=0 \quad \text { for all } x^{*} \in \Sigma, \quad \text { and }\left\|x_{0}\right\| \leq 1 / M .
$$

Now we are ready to prove Theorem 1.7.

Proof of Theorem 1.7. (i) $\Rightarrow(i i)$. Since $f \in H^{\infty}\left(B_{n}\right), f$ has $K$-limit $f^{*}(\zeta)$ for almost every $\zeta \in S$ and $\|f\|_{\infty}=\left\|f^{*}\right\|_{\infty}$. For instance, see Rudin[9]. Thus, for given $\epsilon>0$, we have $\left|f^{*}(\zeta)\right|>\|f\|_{\infty}-\epsilon$ on a subset of $S$ with positive measure. Now, the hypothesis $(i)$ ensures that $\sup _{k}\left|f\left(\alpha_{k}\right)\right| \geq\|f\|_{\infty}$. This proves (ii).

For $(i i) \Rightarrow(i i i)$, it suffices to show that if $\alpha_{0} \neq \alpha_{k}, k=1,2, \cdots$, and $\epsilon>0$ is given, then there exists $\left\{a_{k}\right\}_{k=1}^{\infty}$ such that

$$
e^{\alpha_{0} z}=\sum_{k=1}^{\infty} a_{k} e^{\alpha_{k} z}, \quad \text { and } \sum_{k=1}^{\infty}\left|a_{k}\right|<1+\epsilon .
$$

Define the map

$$
\begin{aligned}
\varphi: H^{\infty}\left(B_{n}\right) & \rightarrow l^{\infty} \\
f & \mapsto\left\{f\left(\alpha_{k}\right)\right\}_{k=0}^{\infty} .
\end{aligned}
$$

From the hypothesis $(i i)$, it is easy to see that $\varphi$ is an isometric embedding and $F=\varphi\left(H^{\infty}\left(B_{n}\right)\right)$ is a norm closed subspace of $l^{\infty}$.

We claim that $F$ is actually weak*-closed. Let $\left\{x_{i}^{*}\right\}$ be a weak*-convergent sequence in $F$. Hence, there is a $M>0$ and $x^{*} \in l^{\infty}$ such that $\left\|x_{i}^{*}\right\|_{\infty} \leq M$ for all $i$ and $\lim _{i \rightarrow \infty} x_{i k}^{*}=x_{k}^{*}$ for $k \in\{0\} \cup \mathbb{N}$, where $x_{i}^{*}=\left(x_{i 0}^{*}, x_{i 1}^{*}, \cdots, x_{i k}^{*}, \cdots\right)$ and $x^{*}=\left(x_{0}^{*}, x_{1}^{*}, \cdots, x_{k}^{*}, \cdots\right)$. Clearly, $x^{*}$ is the weak*-limit of $\left\{x_{i}^{*}\right\}$. We need to show that $x^{*} \in F$. 
Since $\varphi$ is an isometric embedding, there exists a sequence of functions $\left\{f_{i}\right\}$ in $H^{\infty}\left(B_{n}\right)$ such that $\varphi\left(f_{i}\right)=x_{i}^{*}$. Still from the hypothesis $(i i)$, this implies that $\left\|f_{i}\right\|_{\infty} \leq M$ for all $i$ and $\lim _{i \rightarrow \infty} f_{i}\left(\alpha_{k}\right)=x_{k}^{*}$ for $k \in\{0\} \cup \mathbb{N}$. Also from (ii), we see that $\left\{\alpha_{k}\right\}, k \in\{0\} \cup \mathbb{N}$, is a set of uniqueness for $H^{\infty}\left(B_{n}\right)$. Thus, by Vitali's theorem, for instance, see Narasimhan[8], $\left\{f_{i}\right\}$ converges uniformly on compact subsets of $B_{n}$ to a function $f \in H^{\infty}\left(B_{n}\right)$. It follows that $\varphi(f)=x^{*} \in F$. This proves the claim.

Now, we consider the element $x_{0}^{*}=(1,0,0, \cdots) \in l^{\infty} \backslash F$. Since $f \equiv \frac{1}{2} \in$ $H^{\infty}\left(B_{n}\right)$, we get $\operatorname{dist}\left(x_{0}^{*}, F\right) \leq \frac{1}{2}$. If $\operatorname{dist}\left(x_{0}^{*}, F\right)<\frac{1}{2}$, then there is a function $h \in H^{\infty}\left(B_{n}\right)$ such that $\left|h\left(\alpha_{0}\right)-1\right| \leq \frac{1}{2}-\eta,\left|h\left(\alpha_{k}\right)\right| \leq \frac{1}{2}-\eta$ for all $k \in \mathbb{N}$ and some small $\eta>0$. Obviously, this violates the hypothesis $(i i)$. Thus, we have $\operatorname{dist}\left(x_{0}^{*}, F\right)=\frac{1}{2}$.

It follows that, given $\epsilon>0$, one may apply Theorem 2.6 to get an element $x_{0}=\left(a_{0}, a_{1}, a_{2}, \cdots\right) \in l^{1}$ satisfying

(a) $\sum_{k=0}^{\infty} a_{k} f\left(\alpha_{k}\right)=0, \quad$ for all $f \in H^{\infty}\left(B_{n}\right)$,

(b) $a_{0}=1$,

(c) $\sum_{k=0}^{\infty}\left|a_{k}\right|<2+\epsilon$. In particular, $\sum_{k=1}^{\infty}\left|a_{k}\right|<1+\epsilon$.

Property (a) shows that the measure $\mu=\sum_{k=0}^{\infty} a_{k} \delta\left(\alpha_{k}\right)$, where $\delta\left(\alpha_{k}\right)$ is the Dirac unit mass measure at $\alpha_{k}$, satisfies (3) of Lemma 2.3. Hence, it must also satisfy (1) of Lemma 2.3, i.e.,

$$
e^{\alpha_{0} z}+\sum_{k=1}^{\infty} a_{k} e^{\alpha_{k} z}=0 .
$$

Clearly, this proves $($ iii $)$.

To prove $(i i i) \Rightarrow(i v)$, choose an $\alpha_{0}=\left(\alpha_{01}, \cdots, \alpha_{0 n}\right) \in B_{n}$ such that $\alpha_{0 j} \neq$ $\alpha_{k j}$ for all $k \in \mathbb{N}$ and $1 \leq j \leq n$. This is possible since $\left\{\alpha_{k}\right\}$ is countable. Now, according to $(i i i)$, there exists an element $\left(a_{1}, a_{2}, \cdots\right) \in l^{1}$ such that

$$
e^{\alpha_{0} z}=\sum_{k=1}^{\infty} a_{k} e^{\alpha_{k} z} .
$$

This implies that $0<\sum_{k=1}^{\infty}\left|a_{k}\right|<\infty$ and that the measure $\mu=-\delta\left(\alpha_{0}\right)+$ $\sum_{k=1}^{\infty} a_{k} \delta\left(\alpha_{k}\right)$ satisfies (1) of Lemma 2.3. Therefore, if we apply (2) of Lemma 2.3 to the function $f(w)=w_{1}-\alpha_{01}$, we obtain

$$
0=\sum_{k=1}^{\infty} a_{k}\left(\alpha_{k 1}-\alpha_{01}\right) e^{\alpha_{k} z}
$$


Since $\alpha_{k 1}-\alpha_{01} \neq 0$ for all $k \in \mathbb{N}$, we have $0<\sum_{k=1}^{\infty}\left|a_{k}\left(\alpha_{k 1}-\alpha_{01}\right)\right|<\infty$. Thus, (1.1) holds. By an unitary transformation, the second assertion follows from Theorem 1.5. This proves $(i v)$ and the theorem.

Theorem 2.7. $\left(E\left(B_{n}\right),\|\cdot\|_{E}\right)$ is a Banach space, and the dual space of $E\left(B_{n}\right)$ is $H^{\infty}\left(B_{n}\right)$.

Proof. First, choose a sequence of points $\left\{\alpha_{k}\right\}$ in $B_{n}$ such that $(i)$ of Theorem 1.7 is satisfied. Then, by $(i i i)$ of Theorem 1.7 , every function $f \in E\left(B_{n}\right)$ can be represented by using only the exponents $\left\{\alpha_{k}\right\}$, and the norm is not increased. Define

$$
N=\left\{\left(a_{1}, a_{2}, \cdots\right) \in l^{1} \mid \sum_{k=1}^{\infty} a_{k} e^{\alpha_{k} z}=0\right\} .
$$

It is not hard to see that $N$ is a closed subspace of $l^{1}$ and $E\left(B_{n}\right) \simeq l^{1} / N$. Therefore, $\left(E\left(B_{n}\right),\|\cdot\|_{E}\right)$ is a Banach space.

To show $E\left(B_{n}\right)^{*} \simeq H^{\infty}\left(B_{n}\right)$, we first observe that

$$
\left(l^{1} / N\right)^{*}=N^{\perp}=\left\{y^{*} \in l^{\infty} \mid y^{*}(x)=0 \text { for all } x \in N\right\} .
$$

Denote also by $F=\varphi\left(H^{\infty}\left(B_{n}\right)\right)$ as in the proof of $(i i) \Rightarrow($ iii $)$ of Theorem 1.7. Thus, following from Lemma 2.3, we have $F \subset N^{\perp}$. Since $F$ is weak*-closed and weak*-dense in $N^{\perp}$, we actually have $F=N^{\perp}$. Finally, from the choice of $\left\{\alpha_{k}\right\}$ and Theorem 1.7, $\varphi$ is an isometric embedding, and we obtain $E\left(B_{n}\right)^{*} \simeq H^{\infty}\left(B_{n}\right)$.

Let $f \in E\left(B_{n}\right)$ and $g \in H^{\infty}\left(B_{n}\right)$. With $\left\{\alpha_{k}\right\}$ chosen as in Theorem 2.7, if we represent $f=\sum_{k=1}^{\infty} a_{k} e^{\alpha_{k} z}, \sum_{k=1}^{\infty}\left|a_{k}\right|<\infty$, then the pairing asserted in Theorem 2.7 is clearly given by

$$
(f, g)=\sum_{k=1}^{\infty} a_{k} g\left(\alpha_{k}\right)
$$

It follows that

$$
\|f\|_{E}=\sup |(f, g)|
$$

where the supremum is taken over all $g \in H^{\infty}\left(B_{n}\right)$ with $\|g\|_{\infty} \leq 1$. Obviously, it is equivalent to the supremum taken over all $g \circ \varphi$, where $g \in H^{\infty}\left(B_{n}\right)$ with $\|g\|_{\infty} \leq 1$ and $\varphi$ is an automorphism of $B_{n}$.

Now, using the results in one variable (see [2]) and (2.8), (2.9), we can also compute the norm of some interesting examples in $E\left(B_{n}\right)$ as follows:

(1) $\left\|e^{\alpha z}\right\|_{E}=1$, for every $\alpha \in B_{n}$, 
(2) $\left\|e^{\alpha z}-1\right\|_{E}=\frac{2|\alpha|}{1+\sqrt{1-|\alpha|^{2}}}$, for every $\alpha \in B_{n}$,

(3) $\left\|e^{\beta z}-e^{\gamma z}\right\|_{E}=\frac{2\left|\varphi_{\gamma}(\beta)\right|}{1+\sqrt{1-\left|\varphi_{\gamma}(\beta)\right|^{2}}}=\frac{2\left|\varphi_{\beta}(\gamma)\right|}{1+\sqrt{1-\left|\varphi_{\beta}(\gamma)\right|^{2}}}$, for every $\beta, \gamma \in B_{n}$.

Here, for every $\alpha \in B_{n}, \varphi_{\alpha}(z)$ defined by

$$
\varphi_{\alpha}(z)=\frac{\alpha-P_{\alpha} z-\left(1-|\alpha|^{2}\right)^{1 / 2}\left(I-P_{\alpha}\right) z}{1-\langle z, \alpha\rangle}
$$

is an involutive automorphism of $B_{n}$ with the properties: $\varphi_{\alpha}(0)=\alpha$ and $\varphi_{\alpha}(\alpha)=$ 0 . The operator $P_{\alpha}$ is the orthogonal projection of $\mathbb{C}^{n}$ onto the subspace $[\alpha]$ generated by $\alpha$. For details concerning the automorphism $\varphi_{\alpha}(z)$ of $B_{n}$, see Rudin [9].

Proof of (1). Obviously, $\left\|e^{\alpha z}\right\|_{E} \leq 1$. Since, for any $f \in E\left(B_{n}\right),|f(0)| \leq\|f\|_{E}$, we have $1 \leq\left\|e^{\alpha z}\right\|_{E}$. This proves (1).

Proof of (2). Choose an unitary transformation $U$ that maps $\alpha$ to the $j$-th coordinate and apply the result proven in [2] for one variable to the $z_{j}$ variable, we obtain from (2.9)

$$
\begin{aligned}
\left\|e^{\alpha z}-1\right\|_{E} & =\sup _{\substack{g \in H^{\infty}\left(B_{n}\right) \\
\|g\|_{\infty} \leq 1}}|g(\alpha)-g(0)| \\
& =\sup _{\substack{g \in H^{\infty}\left(B_{n}\right) \\
\|g\|_{\infty} \leq 1}}|g \circ U(\alpha)-g \circ U(0)| \\
& =\frac{2|U(\alpha)|}{1+\sqrt{1-|U(\alpha)|^{2}}}=\frac{2|\alpha|}{1+\sqrt{1-|\alpha|^{2}}} .
\end{aligned}
$$

This proves (2).

Proof of (3). From (2) and (2.9), we have

$$
\begin{aligned}
\left\|e^{\beta z}-e^{\gamma z}\right\|_{E} & =\sup _{\substack{g \in H^{\infty}\left(B_{n}\right) \\
\|g\|_{\infty} \leq 1}}|g(\beta)-g(\gamma)| \\
& =\sup _{\substack{g \in H^{\infty}\left(B_{n}\right) \\
\|g\|_{\infty} \leq 1}}\left|g \circ \varphi_{\gamma}(\beta)-g \circ \varphi_{\gamma}(\gamma)\right| \\
& =\left\|e^{\varphi_{\gamma}(\beta) z}-1\right\|_{E} \\
& =\frac{2\left|\varphi_{\gamma}(\beta)\right|}{1+\sqrt{1-\left|\varphi_{\gamma}(\beta)\right|^{2}}} .
\end{aligned}
$$

Since $\left|\varphi_{\beta}(\gamma)\right|=\left|\varphi_{\gamma}(\beta)\right|$ for every $\beta, \gamma \in B_{n}$, thus it is irrelevant to choose $\varphi_{\beta}$ or $\varphi_{\gamma}$. This proves (3). 


\section{Bounded Real HaRmonic Functions on $\Re B_{n}$}

In this section we shall consider the dominating phenomenon of the space of bounded real harmonic functions, denoted by $h^{\infty}\left(\Re B_{n}\right)$, on the open unit ball $\Re B_{n}$ in $\mathbb{R}^{n}, n \geq 3$. Still we denote the boundary of $\Re B_{n}$ by $S$.

The Poisson kernel $P(x, y)$ on $\Re B_{n}$ is defined by

$$
P(x, y)=\frac{1-|x|^{2}}{|x-y|^{n}}, \quad x \in \Re B_{n}, y \in S .
$$

The Poisson integral of a measure $\mu$ on $S$ defined by

$$
P[\mu](x)=\int_{S} P(x, y) d \mu(y)
$$

is a harmonic function in $\Re B_{n}$.

The main effort of this section is to prove the following result which is asserted in the introduction concerning the dominating phenomenon of bounded real harmonic functions on $\Re B_{n}$.

Theorem 3.1. Let $\left\{\alpha_{k}\right\}_{k=1}^{\infty}$ be a sequence of distinct points in $\Re B_{n}, n \geq 3$, with no interior limit points. Then the following three statements are equivalent:

(i) $\left\{\alpha_{k}\right\}$ is nontangentially dense almost everywhere on $S$.

(ii) $\|f\|_{\infty}=\sup _{k}\left|f\left(\alpha_{k}\right)\right|$ for every $f \in h^{\infty}\left(\Re B_{n}\right)$.

(iii) $\left\{\alpha_{k}\right\}$ is a dominating set for $h^{\infty}\left(\Re B_{n}\right)$.

Proof. $(i) \Rightarrow(i i)$. Suppose $f \in h^{\infty}\left(\Re B_{n}\right)$, then there is an $f^{*} \in L^{\infty}(\sigma)$ such that $f$ has nontangential limit $f^{*}(\zeta)$ at almost every boundary point $\zeta \in S$. Here $\sigma$ is the normalized Lebesgue measure on $S$. Thus, by the same argument that proves (ii) from $(i)$ in Theorem 1.7, we are done.

$(i i) \Rightarrow(i)$. Suppose $(i)$ does not hold. Then there is a measurable subset $\Sigma$ of $S$ with positive measure such that every boundary point $y \in \Sigma$ can not be approached nontangentially by $\left\{\alpha_{k}\right\}$.

Now, attach an open regular cone of fixed size to each $y \in \Sigma$ so that the cone is symmetric with respect to the radius $y$. We may assume that the cone is obtained by revolving around the radius $y$ a half line emitted from $y$ which forms a fixed angle $\theta, 0<\theta<\frac{\pi}{2}$, with the radius $y$. Therefore, using this fixed open cone, a truncated open regular cone with height $h \in \mathbb{Q}$, denoted by $C(y, h)$, can be attached to each vertex $y \in \Sigma$ so that $C(y, h) \cap\left\{\alpha_{k}\right\}=\emptyset$. Obviously, we may assume that $0<h \leq h_{0}$ for some fixed small $h_{0} \in \mathbb{Q}$.

Next, for each $h \in \mathbb{Q}, 0<h \leq h_{0}$, denote by

$$
\Sigma_{h}=\left\{y \in \Sigma \mid C(y, h) \cap\left\{\alpha_{k}\right\}=\emptyset\right\} .
$$


We claim that $\Sigma_{h}$ is closed in $\Sigma$. Let $\left\{y_{i}\right\}$ be a sequence in $\Sigma_{h}$ that converges to a $y \in \Sigma$. If $y \notin \Sigma_{h}$, then $C(y, h)$ contains some $\alpha_{k}$. Clearly, this $\alpha_{k}$ will lie in $C\left(y_{i}, h\right)$ for some large $i$. It leads to a contradiction. Hence, $\Sigma_{h}$ is closed in $\Sigma$. In particular, $\Sigma_{h}$ is measurable.

Hence, we have

$$
\Sigma=\underset{\substack{h \in \mathbb{Q} \\ 0<h \leq h_{0}}}{\cup}
$$

It follows that, for some $h \in \mathbb{Q}, 0<h \leq h_{0}, \Sigma_{h}$ has positive Lebesgue measure. We shall denote $F=\Sigma_{h}$ for this $h$. Note that the open region $\cup_{y \in F} C(y, h)$ contains no points from $\left\{\alpha_{k}\right\}$.

Let $y^{*}$ be a point of density of $F$ such that $P\left[\chi_{F}\right](x)$ has nontangential limit 1 at $y^{*}$. Choose a suitable $r \ll h$ such that $\sigma\left(B_{n-1}\left(y^{*}, r\right) \cap F\right) \geq \frac{1}{2} \sigma\left(B_{n-1}\left(y^{*}, r\right)\right)$, where $B_{n-1}\left(y^{*}, r\right)=B_{n}\left(y^{*}, r\right) \cap S$. Now, we just consider the behavior of $P\left[\chi_{F}\right](x)$ on the points $\left\{\alpha_{k}\right\}$ that lie in the open region $\Omega$ between $B_{n-1}\left(y^{*}, r\right)$ and the hyperplane passing through the boundary of $B_{n-1}\left(y^{*}, r\right)$. For each such $\alpha_{k} \in \Omega, \alpha_{k} \notin \cup_{y \in F} C(y, h)$, let $y_{k}=\alpha_{k} /\left|\alpha_{k}\right|$. It is not hard to see that there also exists $r_{k}$ such that

$$
B_{n-1}\left(y_{k}, r_{k}\right) \subset S \backslash F
$$

and that, for every $y \in B_{n-1}\left(y_{k}, r_{k}\right)$, the angle formed by the radius $y_{k}$ and $\overline{\alpha_{k} y}$ is less than or equal to $\theta$.

Now, for each such $\alpha_{k} \in \Omega$, we are ready to estimate $P\left[\chi_{F}\right]\left(\alpha_{k}\right)$. Since

$$
\operatorname{dist}\left(\alpha_{k}, \partial B_{n-1}\left(y_{k}, r_{k}\right)\right) \approx r_{k} \approx 1-\left|\alpha_{k}\right|
$$

obviously there is a constant $c>0$ such that

$$
\begin{aligned}
P\left[\chi_{S \backslash F}\right]\left(\alpha_{k}\right) & =\int_{S} P\left(\alpha_{k}, y\right) \chi_{S \backslash F}(y) d \sigma(y) \\
& \geq \int_{S} P\left(\alpha_{k}, y\right) \chi_{B_{n-1}\left(y_{k}, r_{k}\right)}(y) d \sigma(y) \\
& =\int_{B_{n-1}\left(y_{k}, r_{k}\right)} \frac{1-\left|\alpha_{k}\right|^{2}}{\left|\alpha_{k}-y\right|^{n}} d \sigma(y) \\
& >c .
\end{aligned}
$$

This shows

$$
P\left[\chi_{F}\right]\left(\alpha_{k}\right)=1-P\left[\chi_{S \backslash F}\right]\left(\alpha_{k}\right) \leq 1-c
$$

for $\alpha_{k} \in \Omega$.

Finally, for simplicity, we may assume $y^{*}=(1,0, \cdots, 0)$. Let

$$
f(x)=P\left[\chi_{F}\right](x)+x_{1}+1 .
$$


Then $f$ is a bounded real harmonic function on $\Re B_{n}$. Our construction of $f$ shows, for all $k$,

$$
0 \leq f\left(\alpha_{k}\right) \leq \max \left(3-c, 3-\frac{r^{2}}{2}\right)<3=\|f\|_{\infty} .
$$

Obviously, it contradicts the hypothesis $(i i)$. Therefore, $(i i)$ implies $(i)$.

$($ ii $) \Rightarrow($ iii $)$. This is trivial.

$($ iii $) \Rightarrow($ ii $)$. Suppose $(i i)$ does not hold. Then there is a $f \in h^{\infty}\left(\Re B_{n}\right)$ such that $\|f\|_{\infty}>\sup _{k}\left|f\left(\alpha_{k}\right)\right|$. Choose a number $\gamma$ satisfying $\|f\|_{\infty}>\gamma>$ $\sup _{k}\left|f\left(\alpha_{k}\right)\right|$, and let $g(x) \equiv \gamma$ be a constant function. Obviously, the hypothesis (iii) is violated for these two functions $f$ and $g$. Therefore, (ii) must hold. This completes the proof of the theorem.

\section{Bounded Real $\mathcal{M}$-Harmonic Functions on $B_{n}$}

In this final section we shall consider the dominating phenomenon of the space of bounded real $\mathcal{M}$-harmonic functions, denoted by $\mathcal{M} h^{\infty}\left(B_{n}\right)$, on the open unit ball $B_{n}$ in $\mathbb{C}^{n}, n \geq 2$.

First, we recall briefly some facts about $\mathcal{M}$-harmonic functions. Let $f \in$ $C^{2}\left(B_{n}\right)$ and $a \in B_{n}$, we define the operator $\widetilde{\Delta}$ called invariant Laplacian by

$$
(\widetilde{\Delta} f)(a)=\Delta\left(f \circ \varphi_{a}\right)(0),
$$

where $\varphi_{a}$ is the involution defined in (2.10) and $\Delta$ is the ordinary Laplacian. The invariant Laplacian $\widetilde{\Delta}$ commutes with the automorphisms of $B_{n}$.

Definition 4.1. A function $f \in C^{2}\left(B_{n}\right)$ is called $\mathcal{M}$-harmonic if $(\widetilde{\Delta} f)=0$.

Theorem 4.2. Let $u$ be a $C^{2}$ real-valued function defined on $B_{n}$. Then $u$ is the real part of a holomorphic function on $B_{n}$ if and only if $\Delta u=0$ and $\widetilde{\Delta} u=0$ in $B_{n}$.

Definition 4.3. The kernel

$$
P_{C}(z, \zeta)=\frac{\left(1-|z|^{2}\right)^{n}}{|1-\langle z, \zeta\rangle|^{2 n}}, \quad z \in B_{n}, \zeta \in S
$$

is called the Cauchy-Poisson kernel in $B_{n}$.

Using the Cauchy-Poisson kernel, we can define

$$
P_{C}[\mu](z)=\int_{S} P_{C}(z, \zeta) d \mu(\zeta)
$$


the Cauchy-Poisson integral of the measure $\mu$ on $S$.

Theorem 4.5. For every complex Borel measure $\mu$ on $S, P_{C}[\mu](z)$ is a $\mathcal{M}$ harmonic function.

One should note that the Cauchy-Poisson integral in general need not be harmonic function in the ordinary sense.

Theorem 4.6. If $F \subset S$ with $\sigma(F)=m<1$, where $\sigma$ is the normalized Lebesgue measure on $S$, and $\alpha>1$, then there is a constant $c=c(\alpha, m)>0$ such that

$$
P_{C}\left[\chi_{S \backslash F}\right](z) \geq c
$$

for every $z \notin \Omega(F, \alpha)=\cup_{\zeta \in F} D_{\alpha}(\zeta)$.

For details concerning the $\mathcal{M}$-harmonic functions the reader is referred to Rudin[9].

Next, we introduce the notion of dominating sets for $\mathcal{M}$-harmonic functions.

Definition 4.7. A subset $E$ of $B_{n}$ is said to be a dominating set for $\mathcal{M} h^{\infty}\left(B_{n}\right)$ if for every two functions $f, g \in \mathcal{M} h^{\infty}\left(B_{n}\right)$ satisfying $|f(z)| \leq|g(z)|, z \in E$, we have $\|f\|_{\infty} \leq\|g\|_{\infty}$.

Finally, we prove the main result of this section which has been asserted in the introduction as follows.

Theorem 4.8. Let $\left\{\omega_{k}\right\}_{k=1}^{\infty}$ be a sequence of distinct points in $B_{n}, n \geq 2$, with no interior limit points. Then the following three statements are equivalent:

(i) $\left\{\omega_{k}\right\}$ is $K$-dense almost everywhere on $S$.

(ii) $\|f\|_{\infty}=\sup _{k}\left|f\left(\omega_{k}\right)\right|$ for every $f \in \mathcal{M} h^{\infty}\left(B_{n}\right)$.

(iii) $\left\{\omega_{k}\right\}$ is a dominating set for $\mathcal{M} h^{\infty}\left(B_{n}\right)$.

Proof. $(i) \Rightarrow($ ii $)$. Suppose $f \in \mathcal{M} h^{\infty}\left(B_{n}\right)$, then there is an $f^{*} \in L^{\infty}(\sigma)$ such that $f$ has $K$-limit at every Lebesgue point $\zeta$ of $f^{*}$. Thus, by the same argument that proves $(i i)$ from $(i)$ in Theorem 1.7, we are done.

$(i i) \Rightarrow(i)$. The proof of this direction is similar to that in proving Theorem 1.10. Suppose $(i)$ does not hold. Then there is a measurable subset $\Sigma$ of $S$ with positive measure such that every boundary point $\zeta \in \Sigma$ can not be approached within every $D_{\alpha}(\zeta)$ by $\left\{\omega_{k}\right\}$.

Thus, fix an $\alpha>1$ and a rational number $r_{0}<1$ sufficiently close to 1 , we let

$$
D_{\alpha}(\zeta, r)=D_{\alpha}(\zeta) \cap\left\{z \in B_{n}|| z \mid>1-r\right\},
$$


where $\zeta \in S$ and $r \in \mathbb{Q}, r_{0} \leq r<1$. Hence, for each $\zeta \in \Sigma$, there is a $D_{\alpha}(\zeta, r)$ such that $D_{\alpha}(\zeta, r) \cap\left\{\omega_{k}\right\}=\emptyset$.

Then, for $r \in \mathbb{Q}, r_{0} \leq r<1$, denote by

$$
\Sigma_{r}=\left\{\zeta \in \Sigma \mid D_{\alpha}(\zeta, r) \cap\left\{\omega_{k}\right\}=\emptyset\right\} .
$$

We see that $\Sigma_{r}$ is closed in $\Sigma$. In particular, $\Sigma_{r}$ is measurable. We also have

$$
\Sigma=\underset{r \in \mathbb{Q}}{\cup} \Sigma_{r} .
$$

It follows that, for some $r \in \mathbb{Q}, r_{0} \leq r<1, \Sigma_{r}$ has positive Lebesgue measure. We shall denote $F=\Sigma_{r}$ for this $r$. We may assume that $\sigma(F)<1$. Note also that the open region $\cup_{\zeta \in F} D_{\alpha}(\zeta, r)$ contains no points from $\left\{\omega_{k}\right\}$.

Let $\zeta^{*}$ be a point of density of $F$ such that $\left(K-\lim P_{C}\left[\chi_{F}\right]\right)\left(\zeta^{*}\right)=1$. For simplicity, we may assume $\zeta^{*}=(1,0, \cdots, 0)$. Since $\sigma(F)<1$, Theorem 4.6 shows that there is a constant $c>0$ such that

$$
P_{C}\left[\chi_{S \backslash F}\right](z) \geq c
$$

for every $z \notin \Omega(F, \alpha)=\cup_{\zeta \in F} D_{\alpha}(\zeta)$.

Finally, let $x_{1}=\operatorname{Re} z_{1}$. By Theorems 4.2 and 4.5, we consider the bounded $\mathcal{M}$-harmonic function

$$
h(z)=P_{C}\left[\chi_{F}\right](z)+x_{1}+1 .
$$

It is not hard to see that there is a small $\delta>0$ such that

$$
0 \leq h\left(\omega_{k}\right) \leq 3-\delta<3=\|h\|_{\infty}
$$

for all $k$. Obviously, it contradicts the hypothesis $(i i)$. Therefore, $(i i)$ implies $(i)$.

The equivalence between (ii) and (iii) can be obtained as in the proof of Theorem 1.10. The proof of Theorem 1.11 is now complete.

\section{REFERENCES}

1. F. F. Bonsall, Domination of the supremum of a bounded harmonic function by its supremum over a countable set, Proc. Edinburgh Math. Soc., 30 (1987), 471-477.

2. L. Brown, A. Shields and K. Zeller, On absolutely convergent exponential sums, Trans. Amer. Math. Soc., 96 (1960), 162-183.

3. S. C. Chen, On dominating sets for uniform algebra on pseudoconvex domains, Journal of Pure and Applied Mathematics Quarterly, Special issue in honor of J. J. Kohn, in press.

4. N. Danikas and W. K. Hayman, Domination on sets and in $H^{p}$, Results Math., 34 (1998), 85-90. 
5. W. K. Hayman, Domination on sets and in Norm, Contemporary Mathematics, 404 (2006), 103-109.

6. E. Hille and R. S. Phillips, Functional analysis and semi-groups, Amer. Math. Soc. Colloquium Publications, 1957, p. 31.

7. X. Massaneda and P. J. Thomas, Sampling sequences for Hardy spaces of the ball Proc. Amer. Math. Soc., 128(3) (2000), 837-843.

8. R. Narasimhan, Several Complex Variables, University of Chicago Press, Chicago, 1971.

9. W. Rudin, Function Theory in the Unit Ball of $\mathbb{C}^{n}$, Springer-Verlag, New York, 1980.

So-Chin Chen and Cin-Chang Lu

Department of Mathematics,

National Tsing Hua University,

Hsinchu 30043, Taiwan

E-mail: scchen@math.nthu.edu.tw 